\title{
Assessment of boar sperm function in relation to freezing and storage
}

\author{
W. V. Holt and A. Medrano \\ Institute of Zoology, Zoological Society of London, Regent's Park, \\ London NW1 4RY, UK
}

\begin{abstract}
The functions necessary for normal fertilization to occur in vivo or in vitro are examined and a rational approach to identifying the main features of a fertilizing spermatozoon are developed. It is concluded that methods for testing the quality of spermatozoa must probe the dynamic changes experienced by the spermatozoa during capacitation or under stressful incubation conditions. Recent developments in the multivariate analysis of sperm motility data are used to illustrate the success that can be achieved by this approach. Ideally, changes in sperm motility characteristics should be correlated with an assessment of capacitation status. However, until the capacitation status of any individual cell can be clearly defined this will remain problematic.
\end{abstract}

\section{Introduction}

All sperm function tests share the practical constraint that they inevitably operate by measuring parameters in ejaculated or epididymal spermatozoa, but the results are extrapolated forwards in time to predict the outcome of fertilization in vivo or in vitro. Viewing fertilization in its broadest sense, sperm function tests are required to predict the outcomes of numerous processes, many of which are poorly understood. These include efficiency of sperm transport, speed of capacitation, ability to bind to the egg surface, undergo the acrosome reaction, penetrate the zona pellucida and fuse with the oolemma. The developmental competence of the male pronucleus formed from the spermatozoon can be included in this sequence. To compound the difficulty, these attributes can themselves be reduced to a series of integrated molecular interactions, the efficacy of each being dependent upon developmental processes that took place during spermatogenesis and sperm maturation. This could have been several weeks before the time of sperm assessment.

Assessment of stored semen is performed for reasons that include (1) comparison of storage methods under experimentally controlled conditions, (2) identification of males who, if not subfertile under natural conditions, may provide semen that responds poorly to liquid or frozen storage, and (3) routine quality assurance. As these purposes aim mainly to compare animals, ejaculates or individual samples of semen, exact fertility levels are rarely required. In these situations sperm function tests therefore have a very useful role, and provide valid information that can be interpreted reasonably well on a comparative basis.

\section{What Are the Tests and What Do They Measure?}

During storage, whether by cryopreservation or dilution in an extender, a proportion of the spermatozoa lose their capacity to fertilize. This can be through sustaining a major lesion, such as loss of plasma membrane integrity, acrosomal degeneration, or through more subtle processes. The gross damage is relatively easy to distinguish, and many tests have been developed for the purpose. Some depend upon the exclusion of impermeant dyes by intact cells (e.g. propidium iodide or eosin) by intact cells, or the uptake and subsequent differential loss or retention of fluorescent dyes such as carboxyfluorescein or calcein homodimer (Harrison and Vickers, 1990; Johnson et al., 1996). Use of the fluorescent dyes allows sperm viability testing to be performed by both microscopy and flow 


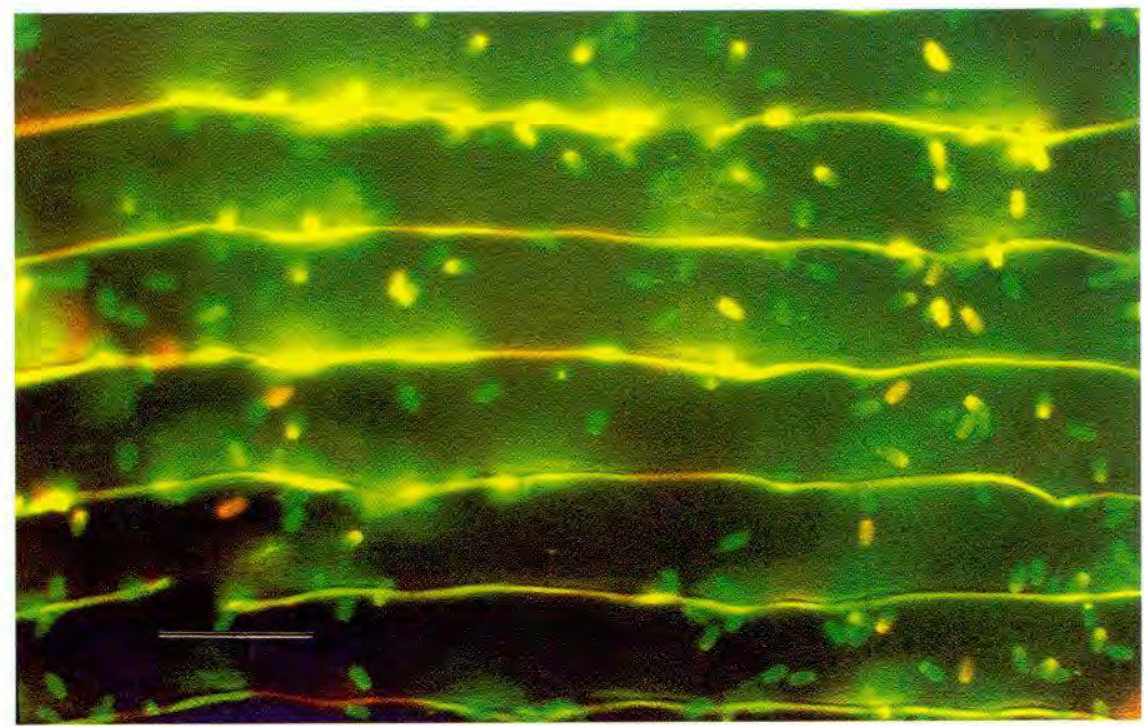

Fig. 1. Photomicrograph showing boar spermatozoa frozen in cryopreservation medium (BF5) on a cryomicroscope stage at $-20^{\circ} \mathrm{C}$. SYBR-14 and propidium iodide were included in the BF5 diluent as probes of membrane integrity. Live cells (SYBR-14 positive) fluoresce green, and dead, or membrane damaged, cells (propidium iodide positive) fluoresce red. Scale bar represents $20 \mu \mathrm{m}$.

cytometry; with the latter technique, integrity of the cell membrane can be monitored quickly on large numbers of cells. One of the fluorescence-based tests, which uses SYBR-14 and propidium iodide to distinguish intact and damaged spermatozoa (Garner and Johnson, 1995), has proved useful for direct observations of spermatozoa in ice during cryomicroscopy (Medrano and Holt, 1996) (Fig. 1). It is also suitable as a long-acting detector of sperm viability within egg yolk-containing media and thus useful for assessing cryopreservation protocols. A different approach to the recognition of membrane-intact spermatozoa involves the hypo-osmotic swelling test (HOS). This is based on the principle that spermatozoa swell in hypotonic media, their flagellae becoming coiled, but only if the plasma membrane remains intact. The HOS test has been combined with the use of fluorescent probes to evaluate acrosomal and plasma membrane integrity simultaneously. Richardson and Aitken (1993) discussed the value of the HOS test in predicting human in vitro fertilization results, and concluded that it does not offer satisfactory predictability relative to the zona-free hamster egg penetration test. While osmotic fragility is likely to vary among individuals within a species, there may be more important species-dependent differences in membrane elasticity. It is therefore inadvisable to extrapolate HOS test conditions between species. Although phase contrast microscopy of fixed spermatozoa has been used extensively to evaluate acrosomal status, generating data about the proportions of normal acrosomal ridges (\% NAR) seen in many publications, this approach has also been largely superseded by the use of fluorescent probes. Many lectins, proteins that interact with the glycoconjugates of the acrosomal membranes or matrix, have been tested for use in acrosomal evaluation (for review, see Cross, 1995). These include peanut agglutinin, Concanavalin A, Pisum sativum and Ricinus communis agglutinins. Monoclonal antibodies are also used for acrosomal evaluation, but in practical terms they are more time consuming (see Cross, 1995). Acrosomal integrity is not necessarily indicative of an intact plasma membrane; therefore, combining two tests for these attributes results in a more discriminatory test than either on its own.

Tests that aim to probe the ability of the cell to respond appropriately to agents that promote the acrosome reaction are now used widely. Methods that test ability to bind and penetrate the zona pellucida also fall into this category. Whitfield and Parkinson (1992) used heparin to induce the acrosome reaction in bull spermatozoa and, in a fertility trial using frozen semen, observed a high 
correlation between 90 day non-return rates and the extent of acrosome reaction induction. This study was remarkable in that the correlation was found even though the acrosome reactions were detected using fresh, not cryopreserved, spermatozoa. One interpretation of this is that freezing itself did not change the heparin responsiveness of the structurally intact cells. Furthermore, the correlation between fresh and frozen spermatozoa also implies that the acrosome reacting ability may be related to capacity to reach the site of fertilization, a function governed mainly by cell motility. Graham and Foote (1987), who used liposomes instead of heparin, found broadly similar results. In human clinical investigations, the principle of examining acrosomal responses to various substances, for example ionophore A23187 and more recently progesterone has been developed, although the validity of this approach has yet to be confirmed. A slightly different strategy for testing the functional integrity of the plasma membrane-acrosome complex has been evaluation of the sperm interaction with isolated zonae pellucidae. As acrosome reactions are induced physiologically by zona pellucida proteins, incubation of spermatozoa with fresh, frozen or salt-stored zonae has been developed as a test. The end-points can be either the number of spermatozoa bound to the zona surface, or the number that undergo the acrosome reaction and commence penetration (see for example, Fazeli et al., 1993). This type of test was developed further with the introduction of the hemi-zona assay (Fazeli et al., 1995), in which one half of a zona is reacted with a reference semen sample while the other is incubated with the sample of interest. Sperm-zona interactions are discussed in more detail by Harrison (this supplement).

If a large proportion of spermatozoa in an inseminate sustains any degree of gross damage, the 'effective concentration' of spermatozoa is reduced, and the number of spermatozoa potentially capable of participating in fertilization is thereby diminished. In some previous reviews (for example Amann, 1989; Den Daas, 1992), the relationship between fertility and number of spermatozoa inseminated has been presented graphically as a dose-response curve with an asymptote, where continued increases in sperm dose do not further increase fertility. Given that for practical purposes the inseminated sperm dose would normally be set within the asymptotic region of the response, reducing the effective concentration of spermatozoa in an inseminate is equivalent to shifting the total sperm concentration back into the sensitive region of the dose-response curve; this could potentially cause a serious reduction in fertility. Assessment of the proportion of grossly intact cells, including those that display normal motility, is thus clearly useful for comparing samples with one another and detecting this type of shift.

Arguments about sperm viability or motility can in principle be extended to include ever more subtle levels of sperm dysfunction; with the application of each new test a subset of spermatozoa can be regarded as non-functional, thereby affecting the dose-response relationship. However, although gross lesions are easily understood, it is more difficult to categorize other differences between individual cells as rendering them unambiguously 'fertile' or 'infertile'. Morphological assessment of spermatozoa provides some clear examples where infertile spermatozoa can be recognized, but this is more applicable to the diagnosis of infertility than to semen assessment from normal donors. For example, occasional cases of human male infertility are attributable to absence of the acrosome or to genetic lesions leading to complete immotility (Kartagener's syndrome). The introduction of highly systematized methods for assessing sperm morphology, the so-called 'strict criteria' (Kruger et al., 1987), may help to consolidate this approach for animal as well as human spermatozoa.

Having recognized that only a proportion of cells survive storage processes with their fertilizing ability intact, the problem of sperm function testing becomes more complex. Two cells may both be intact and responsive, but if one undergoes capacitation and the acrosome reaction before reaching the oocyte, it has rendered itself infertile. On this basis, the definition of fertility becomes very elusive; assuming that an oocyte is already present, the short-lived spermatozoon will be the one that fertilizes. The only conclusion to be drawn from this is that fertility is dependent upon circumstances. Watson (1995) has argued that after freezing and thawing, spermatozoa experience accelerated capacitation which renders them inadequately fertile if they are expected to behave like fresh spermatozoa. If, however, allowed to fertilize within a new shortened time frame, they perform very well. This introduces the concept that sperm assessment should include aspects that test the dynamics of capacitation and fertilization, as well as simply testing the ability of the cell to undergo these 
processes. Viewed in this way, it could be argued that the greatest chances of conception will be associated with semen samples that contain subpopulations of spermatozoa whose dynamics differ; thus the rapidly responding cells will fertilize oocytes if ovulation is early with respect to insemination, while slowly responding cells will be able to remain quiescent and then respond if the interval to ovulation is prolonged.

Regarding sperm function assessment in this way accords well with current concepts of sperm behaviour in the female reproductive tract. Although it has been established that spermatozoa appear within the oviduct within about $15 \mathrm{~min}$ of insemination, these are often a selected population of dead cells carried there by contractions of the female tract. A combination of the intrinsic vigour of the sperm cell and uterine muscular contractions allows only a selected subset of highly motile cells to colonize the uterus and oviduct. To achieve this goal they have to traverse the cervix in species such as cattle, sheep and humans, and/or the uterotubal junction in other species. These processes are highly selective, probably involving aspects of plasma membrane surface biochemistry to allow avoidance of phagocytosis by leucocytes as well as the expression of motility (Harper, 1994). In rodent species it has been shown that traversing the uterotubal junction requires activated, rather than hyperactivated, motility (Olds-Clarke, 1996) emphasizing once more the need for temporal control of capacitation processes.

Once the spermatozoa have reached the oviductal isthmus, it seems that at least a proportion bind to the epithelium and may become quiescent until ovulation (Overstreet and Cooper, 1975; Hunter and Nichol, 1983). The dynamics of this process are poorly understood, but sperm survival is thought to be crucially dependent upon sperm-oviduct interactions. Ovulation itself may provide the trigger for sperm reactivation; the spermatozoa then pass from the isthmus to the ampulla of the oviduct, perhaps using hyperactivated motility to help extricate themselves from oviductal crypts if they become trapped (Suarez, 1987). On reaching the ampulla, the spermatozoa are in principle ready to respond to contact with the oocyte by binding to its surface and undergoing the acrosome reaction. This is another dynamically unstable process, since the spermatozoa can undergo spontaneous acrosome reactions in the absence of an egg. Those that remain intact until receiving a physiological stimulus could, therefore, be considered to constitute the most highly fertile population. Data from recent pig artificial insemination trials lend support to that view; when boar ejaculates were classified as producing large or small litters, large litters were consistently associated with lower frequency of in vitro induced acrosome reactions (Holt et al., 1997).

The above discussion repeatedly identifies the dynamic aspects of sperm function as being important for functional assessment, implying that tests are most informative when they attempt to mimic in vitro various physiological aspects of sperm survival or capacitation. This principle has previously been recognized in some of the older literature that used thermoresistance tests as a crude way of determining whether spermatozoa could survive prolonged heating (Larsson, 1985). The ability to assess capacitation status provides a useful insight into the effects of preservation methods on subsequent sperm dynamics, but there is a need to develop a wider range of techniques. As the topic of sperm function assessment has been reviewed on several occasions recently, the remainder of this review will be confined to considering some of the most recent conceptual advances in sperm motility and capacitation assessment.

\section{Recent Developments in Sperm Motility Analysis}

The value of sperm motion measurements for the diagnosis of human subfertility was demonstrated in the early 1980s (Aitken et al., 1982a,b). However, the manual techniques needed at the time were extremely laborious and consequently were used by few investigators. Computer-assisted semen analysis (CASA) systems have undergone considerable development over the past decade, and now have the capacity to measure multiple motion parameters on hundreds or thousands of individual spermatozoa in any given sample. The value of these systems lies in their ability to perform the motion analyses, and this discussion will concentrate on this aspect of their worth. Mainly in response to clinical demand, they also calculate sperm concentrations and the proportion of motile 
cells; however, they are not ideally suited for this purpose owing to problems of cell versus debris discrimination. Furthermore, although CASA systems are now widely used in clinical laboratories investigating cases of infertility, they are mostly used to make snapshot measurements of sperm behaviour. In view of the arguments presented above which demonstrate the need to measure the dynamics of sperm behaviour, this seems somewhat ill considered.

Studies of human sperm survival in vitro provided a clue to the possibility that examining changes in sperm behaviour is one means of obtaining information relevant to fertility. Sperm swimming speeds in frozen human donor semen samples measured shortly after thawing, and again after incubation for $3.5 \mathrm{~h}$ at $37^{\circ} \mathrm{C}$, revealed that the decline in velocity over that period was associated with differences in fertility when the frozen semen was used in a donor insemination programme (Holt et al., 1989). Higher cumulative pregnancy rates were associated with a greater decline in sperm velocity in vitro. It is important to note that these differences were all derived from measurements of the live, motile, sperm population, indicating that the results cannot be attributed simply to sperm death. This study has since been repeated on a larger scale with similar results (MacLeod and Irvine, 1995).

These studies led us to test the hypothesis that changes in sperm velocity during incubation are correlated with fertility in vivo, this time using the pig as the model system (Holt et al., 1997). Various motion parameters of boar spermatozoa were measured in aliquots of semen prepared for artificial insemination. The semen was transferred from its extender, Beltsville Thawing Solution (BTS), into a Tris-buffered medium containing $40 \mathrm{mmol}$ calcium $\mathrm{H}^{-1}$, incubated at $39^{\circ} \mathrm{C}$ for up to $6 \mathrm{~h}$, and motion parameters measured by CASA. Two separate fertility trials were performed, in which the insemination dose was set to a minimum compatible with commercial AI usage $\left(1.5 \times 10^{9}\right.$ spermatozoa per dose). The high calcium medium was chosen following the demonstration that this medium supported the capacitation of boar spermatozoa, as judged by the zona-free hamster oocyte penetration test (Berger and Horton, 1988). Both fertility trials resulted in significant relationships between sperm velocity during incubation and on-farm fertility, estimated as mean litter size or conception rate. Up to approximately $20 \%$ of variance could be explained by the sperm velocity measurements. In one trial, the correlations with fertility were apparent in the mean velocity parameters over the first $2 \mathrm{~h}$ of incubation, while in the other they were found in the differences in velocity detected over the first $2 \mathrm{~h}$ of incubation. Larger litters and conception rates were associated with increased straight line velocity and track linearity during the first $2 \mathrm{~h}$ in vitro (Fig. 2). Although $20 \%$ of variation seems insignificant in a practical context, it should be remembered that this is against a background where variation is also due to factors such as the skill of the AI operators and differences in female fertility. As these sources of variation become more controlled, the explained variability due solely to semen effects will be relatively increased.

This type of analysis was taken a step further by Moore and Akhondi (1996), who examined rat sperm motility data in relation to in vitro fertilization results. Their studies used an automated CASA system, in which epididymal spermatozoa were incubated in 24-well culture plates and regularly revisited for sampling every $30 \mathrm{~min}$ for $5 \mathrm{~h}$, with the aid of a computer-controlled automatic stage. The fertilizing capacity of spermatozoa correlated exceptionally well with the decline in mean straight line velocity (VSL) over the $5 \mathrm{~h}(r=-0.933 ; P<0.001)$; conversely, there was no relationship between the initial VSL value and fertilizing capacity. The objective of this work was to evaluate an approach that might be useful in reproductive toxicology, where the aim is mainly to find an index of sperm function that reflects events happening during spermatogenesis and sperm maturation; however, this technical approach should also find general application under any circumstances involving inter-sample comparisons.

Under in vitro fertilization conditions there is obviously no sperm selection due to passage through the female tract, so it could be argued that measures obtained with this system are mainly a reflection of the capacity to penetrate the zona pellucida. Although this argument is difficult to refute without further evidence, it is likely that the main attribute being measured is one aspect of a related set of flagellar functions that could include anything from membrane ion permeability and calcium regulation to protein phosphorylation. It would, therefore, be more surprising if in vivo and in vitro studies were found to be completely independent. 


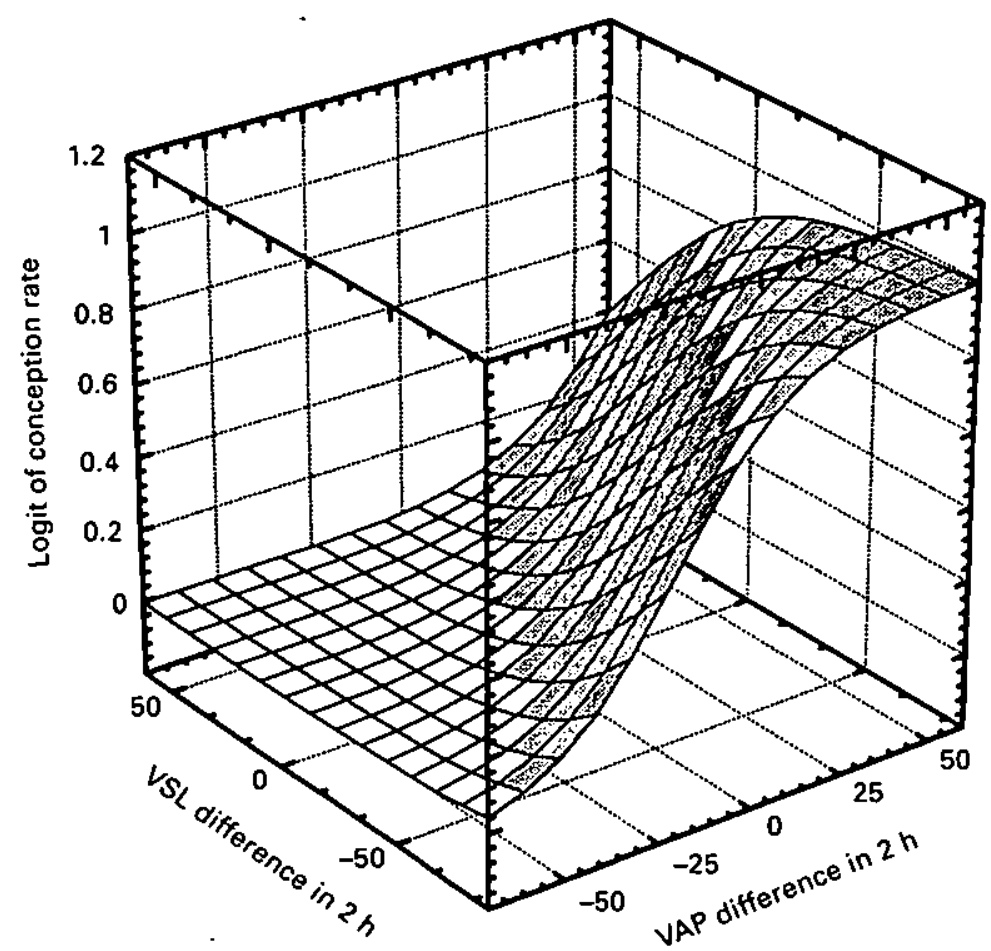

Fig. 2. Three-dimensional plot representing the relationships between conception rate and boar sperm velocity changes during incubation for $2 \mathrm{~h}$ at $39^{\circ} \mathrm{C}$ in Tris-buffered medium, containing $40 \mathrm{mmol}$ calcium $\mathrm{l}^{-1}$. Increases in VSL (straight line velocity) over $2 \mathrm{~h}$, represented as negative differences, and decreases in VAP (average path velocity), represented as positive differences, were associated with higher conception rates (logit transform) in a fertility trial. These data were from a study undertaken in collaboration with the Meat \& Livestock Commission and JSR Healthbred Ltd. For further details see Holt et al. (1997).

In the studies cited above the sperm motion data were always analysed in terms of population mean values; this method of analysis is almost universal in studies using CASA. The main assumption with this approach is that samples of semen contain a homogeneous population of spermatozoa, which tend to behave synchronously throughout their lifetime. However, several lines of evidence would contradict this view; and it is evident that there are a number of sperm subpopulations that behave asynchronously. Perhaps one of the clearest examples of this principle is that of transmission distortion in mice heterozygous for the $\mathrm{t}^{\mathrm{w} \cdot 32}$ gene, where almost all eggs are fertilized in vivo by the $\mathrm{t}^{\mathrm{w} 32}$-carrying spermatozoa, even though the $50 \%$ of spermatozoa that do not carry this gene are normally fertile when tested in vitro. The explanation for this effect probably includes molecular differences in flagellar membrane function between the two sperm subpopulations, which lead to variations in motility and thus differential ability to gain access to the oviduct (Olds-Clarke and Sego, 1992). While this example probably represents an extreme form of subpopulation structure within a semen sample, other examples also occur for which there is currently no known explanation. Sex ratio distortion has been related to the timing of insemination with respect to ovulation (Clutton-Brock et al., 1986), an effect that could be attributable to differences in capacitation rate between $X$ - and $Y$-chromosome bearing spermatozoa.

At present it is not possible to correlate unequivocally the motion parameters of individual spermatozoa with their genetic content; this will require the development of innocuous DNA probes that can be applied to spermatozoa without killing them. However, the wealth of multivariate motion 
data available from CASA systems has stimulated interest in the problem of characterizing sperm subpopulations on the basis of their motility. Recognition of hyperactivated human spermatozoa as a discrete subset has been explored by several research groups (see Irvine, 1995), some of which have then used their criteria of hyperactivation as the basis for correlating the proportion of hyperactivated cells in an ejaculate with various fertility outcomes. This approach seems very promising, and in some cases the incidence of hyperactivation has proved highly predictive of in vitro fertilization rate. Sukcharoen et al. (1996) could correctly predict over $70 \%$ of good or poor IVF results using hyperactivation criteria alone. This approach to the identification of sperm subsets in semen was also used by Davis et al. (1995) to see whether characteristics of the sperm population substructure in fresh samples could be used to predict how well the samples would survive cryopreservation. This study is especially interesting as it provides statistical methodology for the objective identification of subsets by cluster analysis, but also because it was concluded that a small set of kinematic variables measured in fresh samples could be used to predict the proportion of motile cells present after freezing and thawing. The interpretation of this somewhat surprising result is presumably that the motion parameters of the live cells measured before freezing are identifying subpopulations that are particularly resistant or vulnerable to the freeze-thawing process. The authors commented that their best overall regression model predicted motility from the difference in straight line velocity (VSL) between the cluster with the highest VSL and the cluster with the lowest VSL.

These novel approaches to the use of CASA have been explored to a much lesser extent with animal spermatozoa. Holt (1996) outlined cluster analysis approaches to the identification of sperm subsets within boar semen samples. It was shown that when bicarbonate and caffeine were used to induce different types of sperm motion, the changing population substructures could also be detected numerically. Earlier it had also been demonstrated that if artificial data sets were constructed by combining measurements from three sperm populations showing different types of movement, defined as circular, progressive or erratic, cluster analysis algorithms could disentangle the data with reasonable efficiency (Holt et al., 1992).

All fertility trials conducted in animal species use either homospermic or heterospermic insemination protocols. In the latter case, semen samples from two or more individuals are mixed prior to insemination. Correlations of CASA data with fertility results have been attempted within both paradigms; in some cases marked differences in the outcomes of these correlations have been observed. When Budworth et al. (1988) studied frozen-thawed bull semen, no significant relationships between CASA parameters and homospermic inseminations were detected (all $r$ values $<0.1$ ), but the motion parameters curvilinear velocity (VCL) and straightline velocity (VSL) accounted for approximately $70 \%$ of variance in competitive fertility index under the heterospermic insemination protocol. Such contrasting relationships must point to fundamental differences between the biology of the two systems, and suggest that the two types of test are addressing different questions. With homospermic inseminations the question is 'will conception occur, and if so how many eggs will be fertilized?', while for heterospermic inseminations there is an implicit assumption that conception will definitely occur and the question is then 'which subpopulation of sperm is more successful?'. This is similar to the analysis presented above where there are also subpopulations within a single sample of semen. As the questions are different, the answers can hardly be the same. The arguments presented here strongly indicate that although the technology for measuring sperm motion parameters is now well developed (see, Boyers et al., 1989; Davis and Katz, 1992, 1993), much is yet to be done on methods of data analysis. Exploration of the multivariate analysis approach combined with dynamic aspects of sperm motility is likely to be the best option for extracting useful information from the use of CASA.

Integrating such approaches into the routine working environment of a commercial pig AI centre, where hundreds of semen samples have to be collected and diluted for efficient distribution to farmers, may require some compromises to be made. The time delay introduced by preparing semen samples for CASA, and the need for repeat assessments over 1 or $2 \mathrm{~h}$, will obviate use of the technique as a routine screening method for all ejaculates. However, the method is likely to be useful in planned quality assurance programmes or for testing the effects of modified practices upon the quality of stored semen. 


\section{Developments in the Detection of Capacitation}

Ideally, it would be useful to combine the measurement of sperm motion with assessment of capacitation status. This approach would allow us to answer the type of questions recently raised by Watson (1995), who proposed that cryopreservation causes accelerated capacitation and consequently shortens the longevity of spermatozoa in vivo and in vitro. Watson (1995) reviewed evidence from in vitro studies of human and ram semen, both of which supported the principle that frozen-thawed spermatozoa were able to fertilize oocytes almost immediately, whereas fresh spermatozoa required an interval of preincubation before acquiring the same readiness to fertilize. Similar arguments can be adduced from in vivo studies, where the lifespan of frozen-thawed spermatozoa in the female tract has been demonstrated to be considerably shorter than that of fresh semen. Whether this is an artificial form of destabilization, rather than a true manifestation of capacitation, requires further research. For example, it is known that capacitation is accompanied by membrane hyperpolarization (Zeng et al., 1995), which possibly permits the spermatozoa to switch rapidly between hyperactivated and activated motility, and allows them to respond appropriately to the zona pellucida stimulus.

Current concepts of capacitation were reviewed recently by Harrison (1996), who confirmed that individual spermatozoa within a population become capacitated at different rates. For studies of sperm dynamics, precise knowledge of capacitation in individual cells is crucial, as it would permit characterization of the sperm population behaviour in great detail. To date there are no tests that discriminate microscopically between capacitated and non-capacitated spermatozoa; this is partly because recognizing that an individual spermatozoon has moved from the uncapacitated to the capacitated state requires foreknowledge of its ability to fertilize the oocyte. Most data relevant to this problem have come from flow cytometric studies, observation of responses to the zona pellucida or calcium ionophore stimulus, or through the application of a fluorescent antibiotic, chlortetracycline (CTC). However, all of this is circumstantial, and based on the behaviour of sperm populations rather than of individuals. The interesting aspect of CTC is that individual cells can be distinguished on the basis of sperm head fluorescence patterns. These have been considered as (1) uncapacitated (F pattern), (2) acrosome reacted (AR) or (3) B pattern, where the fluorescence is diminished over part of the sperm head; the $B$ pattern has been widely interpreted as indicating capacitation (Wang et al., 1995). Watson (1996) showed that cooling boar spermatozoa induced an increased incidence of $B$ patterns, thus supporting the contention that cryopreservation accelerates capacitation. The exact mechanism of CTC staining remains controversial; one explanation is that it may interact with calcium at the sperm surface, and thereby be indicative of calcium ATPase activity (Adeoya-Osiguwa and Fraser, 1996). So far, experiments involving CTC have involved fixation of the spermatozoa before their evaluation; this necessarily precludes the simultaneous assessment of motility and capacitation status, al though such an approach would be highly informative.

It seems self evident that any spermatozoon that fertilizes an egg must have been through the capacitation process; however, recognizing the process unequivocally may be impossible as any test has, by definition, to be predictive and therefore subject to uncertainty. The problem is hampered by the likelihood that at any given moment, only a small proportion of spermatozoa have the ability to fertilize. As hyperactivation is at least a functional property required for zona penetration, the efficient recognition of this attribute may ultimately prove more effective than the search for visible changes in plasma membrane organization.

\section{Conclusions}

In this review we take the position that rather than listing a series of sperm function tests and their technical merits, it is more important and interesting to analyse the meaning of such tests and from that discussion derive arguments that guide future research. The most important conclusions from this exercise seem to be that sperm function testing is not always aimed at estimating fertility on a quantitative basis, and that having realized this it is possible to derive a considerable amount of 
comparative information of experimental or practical value. The overriding aspect of the discussion is that as more becomes known about the dynamic changes undergone by spermatozoa during physiological processes such as capacitation, it will be possible to derive ever better objectively based function tests. The disadvantage of the logical approach to sperm function tests is that they may become too complex for implementation in routine laboratories, where the aim is quality assurance rather than diagnosis or research. It will be a challenge to simplify the available tests without losing sight of the requisite functional assessments.

\section{References}

Adeoya-Osiguwa SA and Fraser LR (1996) Evidence for Ca2*dependent ATPase activity, stimulated by decapacitation factor and calmodulin, in mouse sperm Molectular Reproduction and Development $44111-120$

Aitken RJ, Best FSM, Richardson DW, Djahanbakhch $O$ and Lees MM (1982a) The correlates of fertilizing capacity in normal fertile men Fertility and Sterility 38 68-76

Aitken RJ, Best FSM, Richardson DW, Djahanbakhch, $O$ and Templeton A (1982b) An analysis of semen quality and sperm function in cases of oligospermia Fertility and Sterility 38 705-711

Amann RP (1989) Can the fertility potential of a seminal sample be predicted accurately? Journal of Andrology 10 89-98

Berger T and Horton MB (1988) Evaluation of assay conditions for the zona-free hamster bioassay of boar sperm fertility Gamete Research 19 101-111

Boyers SP Davis R and Katz DF (1989) Automated semen analysis Current Problems in Obstetrics and Gymaecology 12 172-200

Budworth PR, Amann RP and Chapman PL (1988) Relationships between computerized measurements of motion of frozen-thawed bull spermatozoa and fertility fournal of Andrology 9 41-54

Clutton-Brock TH, Albon SD and Guinness FE (1986) Great expectations - dominance, breeding success and offspring sex-ratios in red deer Animal Behaviour 34 460-471

Cross N (1995) Methods for evaluating the acrosomal status of human sperm. In Human sperm acrosome reaction pp 277-285 Eds P Fenichel and J Parinaud. John Libby Eurotext Ltd, Montrouge

Davis RO and Katz DF (1992) Standardization and comparability of CASA instruments Joumal of Andrology 13 81-86

Davis RO and Katz DF (1993) Computer-aided sperm analysis: technology at a crossroads Fertility and Sterility 59 953-955

Davis RO, Drobnis EZ and Overstreet JW (1995) Application of multivariate cluster, discriminate function, and stepwise regression analyses to variable selection and predictive modeling of sperm cryosurvival Fertility and Sterility 63 1051-1057

Den Daas N (1992) Laboratory assessment of semen characteristics Animal Reproduction Science 28 87-94

Fazeli AR, Steenweg W, Bevers, MM, De Loos FAM, Van den Brock $J$ and Colenbrander B (1993) Development of a sperm zona pellucida binding assay for bull sperm Veterinary Record 132 14-16

Fazeli AR, Holt C, Steenweg W, Bevers MM, Holt WV and Colenbrander B (1995) Development of a sperm hemizona binding assay for boar semen Theriogenology 44 17-27

Gamer DL and Johnson LA (1995) Viability assessment of mammalian sperm using SYBR-14 and propidium iodide Biology of Reproduction 53 276-284

Graham JK and Foote RH (1987) Dilaurylphosphatidyl-choline liposome effects on the acrosome reaction and in vitro penetration of zona-free hamster eggs by bull spermato zoa: A fertility assay for frozen-thawed semen Gamete Research 16 147-158

Harper MJK (1994) Gamete and zygote transport. In The Physiology of Reproduction pp 123-187 Eds E Knobil and JD Neill. Raven Press, New York

Harrison RAP (1996) Capacitation mechanisms, and the role of capacitation as seen in eutherian mammals Reproduction, Fertility and Development 8 581-594

Harrison RAP and Vickers SE (1990) Use of fluorescent probes to assess membrane integrity in mammalian spermatozoa Joumal of Reproduction and Fertility 88 343-352

Holt C, Moore HDM, Reed HCB and Holt WV (1992) Can sperm subpopulations be recognized within boar semen samples? An approach using cluster analysis Journal of Reproduction and Fertility Abstract Series 10 Abstract 81

Holt C, Holt WV, Moore HDM, Reed HCB and Cumock RM (1997) Objectively measured boar sperm motility parameters correlate with the outcomes of on-fam inseminations. Results of two fertility trials Journal of Andrology 18 20-31

Holt WV (1996) Can we predict fertility rates? Making sense of sperm motility Reproduction in Domestic Animals 31, 17-24

Holt WV, Shenfield F, Leonard T, Hartman TD, North RD and Moore HDM (1989) The value of sperm swimming speed measurements in assessing the fertility of human frozen semen Human Reproduction 4 294-297

Hunter RHF and Nichol R (1983) Transport of spermatozon in the sheep oviduct: preovulatory sequestering of cells in the caudal isthmus fournal of Experimental Zoology 228 121-128

Irvine DS (1995) Computer-assisted semen analysis systems: sperm motility assessment Human Reproduction 10 53-59

Johnson LA, Maxwell WMC, Dobrinsky JR and Welch GR (1996) Staining sperm for viability assessment Reproduction in Donestic Animals 31 37-47

Kruger TF, Acosta AA, Simmons KF, Swanson BJ, Matta JF, Veeck LL, Morshedi M and Brugo S (1987) New method of evaluating sperm morphology with predictive value for human in vitro fertilization Urology 30 248-592

Larsson K (1985) Boar sperm viability after freezing and thawing. In Deep Freezing of Boar Semen pp 177-187 Eds E Knobil and JD Neill. Raven Press, New York

Macleod IC and Irvine DS (1995) The predictive value of computer assisted semen analysis in the context of a donor insemination programme Human Reproduction 10 580-586

Medrano A and Holt WV (1996) Protective effects of glycerol during cold shock in boar spermatozoa: a cryomicroscope study using propidium iodide and Sybr-14 Reproduction in Domestic Animals 31 281-282

Moore HDM and Akhondi MA (1996) Fertilizing capacity of rat spermatozoa is correlated with decline in straight-line velocity measured by continuous computer-aided sperm analysis -epididymal rat spermatozoa from the proximal cauda have 
a greater fertilizing-capacity in-vitro than those from the distal cauda or vas deferens joumal of Andrology 1750-60

Olds-Clarke $P$ (1996) How does poor motility alter sperm fertilizing ability Joumal of Andrology 17 183-186

Olds-Clarke $P$ and Sego R (1992) Calcium alters capacitation and progressive motility of uterine spermatozoa from $+/+$ and congenic $\mathrm{t}^{\mathrm{w} 32 /+}$ mice Biology of Reproduction 47, 629-635

Overstreet JW and Cooper, CW (1975) Reduced sperm motility in the isthmus of the rabbit oviduct Nature 258 718-719

Richardson DW and Aitken RJ (1993) Techniques for examining the fertilizing capacity of spermatozoa. In Donor Insemination pp 50-70 Eds CLR Barratt and ID Cooke. Cambridge University Press, Cambridge

Suarez SS (1987) Sperm transport and motility in the mouse oviduct - observations in situ. Biology of Reproduction 36, 203-210

Sukcharoen N, Keith J, Irvine DS and Aitken RJ (1996) Prediction of the in vitro fertilization (IVF) potential of human spermatozoa using sperm function tests: the effect of the delay between testing and IVF Human Reproduction:11 1030-1034
Wang WH, Abeydeera LR, Fraser LR and Niwa K (1995) Functional-analysis using chlortetracycline fluorescence and in vitro fertilization of frozen-thawed ejaculated boar spermatozoa incubated in a protein-free chemically defined medium Journal of Reproduction and Fertility 104, 305-313

Watson PF (1995) Recent developments and concepts in the cryopreservation of spermatozoa and the assessment of their post-thawing function Reproduction, Fertility and Development $7871-891$

Watson PF (1996) Cooling of spermatozoa and fertilizing capacity Reproduction in Domestic Animals 31 135-140

Whitfield CH and Parkinson TJ (1992) Relationship between fertility of bovine semen and in vitro induction of acrosome reactions by heparin Theriogenology 38 11-20

Zeng Y, Clark EN and Florman HM (1995) Sperm membrane potential-hyperpolarization during capacitation regulates zona pellucida-dependent acrosomal secretion Developmental Biology 171 554-563 